\title{
DEFORMATION RIGIDITY OF THE RATIONAL HOMOGENEOUS SPACE ASSOCIATED TO A LONG SIMPLE ROOT
}

\author{
BY JUN-MUK HWANG ${ }^{1}$ AND NGAIMING MOK ${ }^{2}$
}

ABSTRACT. - As a continuation of our previous works we study the conjecture on the rigidity under Kähler deformation of the complex structure of rational homogeneous spaces $G / P$ of Picard number 1 , confirming its validity whenever $G / P$ is associated to a long simple root. For these rational homogeneous spaces the minimal $G$-invariant holomorphic distribution $D$ is spanned by varieties of minimal rational tangents, and, excepting the symmetric and the contact cases, the complex structure of $G / P$ is completely determined by the nilpotent symbol algebra of the weak derived differential system of $D$. The problem is reduced, in a sense, to the invariance of this nilpotent symbol algebra under Kähler deformation. In our earlier works in relation to the question of the integrability of distributions spanned by varieties of minimal rational tangents we have established identities on Lie brackets using integral surfaces arising from pencils of rational curves. In the case on hand, at a point $o \in G / P$ we prove that the nilpotent symbol algebra at $o$ is nothing other than the universal Lie algebra generated by $D_{o}$ subject to these identities on Lie brackets, by verifying that they correspond to finiteness condition in the Serre presentation of the simple Lie algebra $G$.

(c) 2002 Éditions scientifiques et médicales Elsevier SAS

RÉSUMÉ. - Nous continuons à étudier la conjecture sur la rigidité sous déformation kählérienne de la structure complexe des espaces homogènes rationnels $G / P$ de nombre de Picard égal à 1 , en confirmant sa validité dans le cas où $G / P$ est associé à une racine simple longue. Pour ces espaces homogènes rationnels la distribution $G$-invariante minimale $D$ est engendrée par les variétés de tangentes rationnelles minimales et, à l'exception des variétés symétriques ou de contact, la structure complexe de $G / P$ est complètement déterminée par l'algèbre nilpotente des symboles du système différentiel dérivé faible défini par $D$. Dans un sens le problème est réduit à la vérification de l'invariance de cette algèbre sous déformation kählérienne. Dans nos travaux antérieurs concernant l'intégrabilité des distributions engendrées par des variétés de tangentes rationnelles minimales nous avons établi des identités pour des crochets de Lie en utilisant des surfaces intégrales provenant des pinceaux de courbes rationnelles. Dans le cas présent, à un point $o \in G / P$ nous démontrons que l'algèbre nilpotente des symboles à $o$ n'est rien d'autre que l'algèbre universelle engendrée par $D_{o}$ assujettie à ces identités, en vérifiant que les dernières correspondent aux conditions de finitude dans la présentation de Serre de l'algèbre de Lie simple $G$.

(c) 2002 Éditions scientifiques et médicales Elsevier SAS

As a continuation of our previous works [5] and [3], we study the following conjecture on the rigidity of rational homogeneous spaces of Picard number 1 under Kähler deformation. For the background of this conjecture, see the introduction of [5].

\footnotetext{
${ }^{1}$ Supported by Grant No. 98-0701-01-5-L from the KOSEF.

${ }^{2}$ Supported by a CERG of the Research Grants Council of Hong Kong.
} 
CONJECTURE. - Let $G$ be a complex simple Lie group and $P$ be a maximal parabolic subgroup. Let $\pi: \mathcal{X} \rightarrow \Delta=\{t \in \mathbf{C},|t|<1\}$ be a smooth projective morphism from a complex manifold to the unit disc. If $X_{t}:=\pi^{-1}(t)$ is biholomorphic to $G / P$ for all $t \neq 0$, then $X_{0}$ is also biholomorphic to $G / P$.

A natural approach is to construct a geometric structure on $X_{0}$ using the tangent vectors to minimal rational curves. In [5] (resp. [3]), we constructed a G-structure (resp. a contact structure) this way and proved the Conjecture. By the work of Yamaguchi [16], for the cases different from the symmetric or the contact cases, it suffices to recover the nilpotent Lie algebra structure of a differential system to prove the Conjecture. The purpose of this paper is to show this when $P$ is associated to a long simple root, including the cases of all maximal parabolic subgroups when all roots of $G$ are of the same length:

MAIN THEOREM. - Let $G$ be a complex simple Lie group and $P$ be a maximal parabolic subgroup associated to a long simple root. Let $\pi: \mathcal{X} \rightarrow \Delta=\{t \in \mathbf{C},|t|<1\}$ be a smooth projective morphism from a complex manifold to the unit disc. If $X_{t}:=\pi^{-1}(t)$ is biholomorphic to $G / P$ for all $t \neq 0$, then $X_{0}$ is also biholomorphic to $G / P$.

As in [5] and [3] our approach consists of studying distributions derived from varieties of minimal rational tangents (see Section 2 for the definition), notably on questions of integrability. There is however an essential difference in that we have to deal with a nilpotent Lie algebra structure of the differential system, which is much more complicated than a G-structure or a contact structure. The hypothesis on $P$ enters in a crucial way in the proof. In fact, $P$ is associated to a long simple root if and only if the minimal $G$-invariant distribution on $G / P$ is spanned by varieties of minimal rational tangents.

With some oversimplification to streamline the comparison with earlier works the proof of the Main Theorem breaks down into three steps. The first step, which parallels the first steps of [5] and [3], is to show that the normalized space $\mathcal{K}_{x}$ of minimal rational curves at a generic point $x$ of $X_{0}$ agrees with that of the model $G / P$. The proof of this step is a refinement of arguments in [5] or [3] requiring deeper knowledge of the geometry of Hermitian symmetric spaces (as varieties of minimal rational tangents). The second step is to show that the variety of minimal rational tangents $\mathcal{C}_{x} \subset \mathbf{P} T_{x}\left(X_{0}\right)$, which is the image of $\mathcal{K}_{x}$ under the tangent map, agrees with that of the model as a projective subvariety. The third step is then to show that the differential system generated by the varieties of minimal rational tangents has the same nilpotent Lie algebra structure as that of the model $G / P$. The second and the third steps are closely intertwined and handled together in Section 3. Here new difficulties arise which were not present in [5] or [3]. As a matter of fact, while the third step is completely trivial for the Hermitian symmetric case and is rather straightforward for the contact case, it is highly non-trivial in other cases covered by the Main Theorem. An analogue of the third step is also the main obstacle in extending the Main Theorem to the case when $P$ is associated to a short root.

For the second step, in the Hermitian symmetric case it is enough to show that the varieties of minimal rational tangents at the central fiber span the full tangent bundle; in the contact case it is enough to show that they must span a distribution of codimension 1 (as on a generic fiber). In both cases assuming the contrary we would have obtained an integrable distribution spanned by varieties of minimal rational tangents, leading to a contradiction since $X_{0}$ is of Picard number 1 . Essential to this line of proof is the particular projective geometry of the variety of minimal rational tangents of the model space $G / P$. For instance, in the model contact case, varieties of minimal rational tangents span the contact distribution $D$, and are Legendrian subvarieties of the projectivization of $\mathbf{P} D$. From this it followed that any drop in the rank (when compared to $D$ ) of the distribution $W$ spanned by varieties of minimal rational tangents in the central fiber $X_{0}$ 
would force $W$ to be integrable by results from [5]. In other words, we relied on the fact that the contact distribution in the model contact manifold is just short of being integrable.

In the situation of the Main Theorem and assuming that $G / P$ is neither Hermitian symmetric nor of the contact type, the distribution $D$ spanned by varieties of minimal rational tangents on the model space can be very far away from being integrable. The problem is to prove that the failure of integrability, in a sense to be made precise, is stable under deformation. Even on the model space the differential system may have many levels, and jumps of simple numerical invariants such as ranks of distributions are far from being enough to lead to contradictions. On $X_{0}$ we have to consider the differential system obtained by augmenting $W$ by taking successive Lie brackets. The nilpotent Lie algebra structure associated to the differential system is precisely the algebraic structure in which the failure of integrability is encoded. The novel point of the proof of our Main Theorem is Proposition 6, which shows that a natural integrability condition obtained in [5] coming from the deformation theory of minimal rational curves turns out to be equivalent to the finiteness condition in the Serre presentation of the simple Lie algebra. This is essentially a result on the model $G / P$ and is expected to be useful in the study of geometry of $G / P$ itself, independent of the deformation problem.

In a sense, the main motivation for studying the Conjecture for us is that it is a good testing ground for the study of Fano manifolds of Picard number 1 through minimal rational curves. The problem of recovering the structure of a given Fano manifold of Picard number 1 from the information on the minimal rational curves is broader and of greater importance to us than the Conjecture itself. From this perspective the study of the large classes of $G / P$ in the Main Theorem reveals that the deformation theory of rational curves provides a powerful tool to unravel the algebraic structures of differential systems arising from distributions spanned by varieties of minimal rational tangents. In the case at hand it provides a means of identifying varieties of minimal rational tangents and recovering the complex structure of these rational homogeneous spaces. It is in this context that we believe that our result enhances the general perspective in our geometric study of Fano manifolds as put forth in [5-7].

\section{Rational homogeneous spaces associated to long simple roots}

In this section, we will review some basic facts about the rational homogeneous space associated to a long simple root (see e.g. [16] or Section 2 of [6]).

Let $\mathbf{g}$ be a complex simple Lie algebra. Choose a Cartan subalgebra $\mathbf{h}$ and the root system $\Phi \subset \mathbf{h}^{*}$ of $\mathbf{g}$ with respect to $\mathbf{h}$. Fix a system of simple roots $\left\{\alpha_{1}, \ldots, \alpha_{l}\right\}$ and a distinguished choice of a simple root $\alpha_{i}$. Given an integer $k,-m \leqslant k \leqslant m$, we define $\Phi_{k}$ as the set of all roots $\sum_{q=1}^{l} m_{q} \alpha_{q}$ with $m_{i}=k$. Here $m$ is the largest integer such that $\Phi_{m} \neq 0$. For $\alpha \in \Phi$, let $\mathbf{g}_{\alpha}$ be the corresponding root space. Define

$$
\mathbf{g}_{0}=\mathbf{h} \oplus \bigoplus_{\alpha \in \Phi_{0}} \mathbf{g}_{\alpha}, \quad \mathbf{g}_{k}=\bigoplus_{\alpha \in \Phi_{k}} \mathbf{g}_{\alpha}, \quad k \neq 0 .
$$

The decomposition $\mathbf{g}=\bigoplus_{k=-m}^{m} \mathbf{g}_{k}$ gives a graded Lie algebra structure on $\mathbf{g}$. Define

$$
\begin{aligned}
& \mathbf{p}=\mathbf{g}_{0} \oplus \mathbf{g}_{-1} \oplus \cdots \oplus \mathbf{g}_{-m}, \quad \mathbf{l}=\mathbf{g}_{0}, \\
& \mathbf{u}=\mathbf{g}_{-1} \oplus \cdots \oplus \mathbf{g}_{-m} .
\end{aligned}
$$

We say that $\mathbf{p}$ is the maximal parabolic subalgebra associated to the simple root $\alpha_{i} . \mathbf{u}$ is the unipotent radical of $\mathbf{p}$ and $\mathbf{p}=\mathbf{u}+\mathbf{l}$ is a Levi decomposition. Let us remark that our choice of $\mathbf{p}$ 
has signs of roots different from the choice in some references, e.g., [16]. We prefer this choice because positive roots correspond to positive line bundles.

Each $\mathbf{g}_{j}, 1 \leqslant j \leqslant m$, is an irreducible l-module. Let $W \subset \mathbf{g}_{1}$ be the cone of highest weight vectors of the irreducible l-module $\mathbf{g}_{1}$. Its projectivization $\mathbf{P} W \subset \mathbf{P g}_{1}$ will be called the highest weight variety. 1 has 1 -dimensional center. The semi-simple part of 1 has rank $l-1$ and its Dynkin diagram is obtained by removing $\alpha_{i}$ from the Dynkin diagram of $\mathrm{g}$. From this, one can easily determine the highest weight variety in $\mathbf{P g}_{1}$. We list the pairs $\left(\alpha_{i} ; \mathbf{P} W\right)$ below. For the numbering of simple roots, we will use the convention of [16].

- $\mathbf{g}=A_{l}$

$$
\left(\alpha_{i} ; \mathbf{P}_{i-1} \times \mathbf{P}_{l-i}\right)
$$

- $\mathbf{g}=B_{l}$

$\left(\alpha_{i} ; \mathbf{P}_{i-1} \times \mathbf{Q}_{2(l-i)-1}\right)$ for $1 \leqslant i \leqslant l-1,\left(\alpha_{l} ; G r(2, l-2)\right)$

- $\mathbf{g}=C_{l}$

$\left(\alpha_{i} ; \mathbf{P}_{i-1} \times \mathbf{P}_{2(l-i)-1}\right)$ for $1 \leqslant i \leqslant l-1,\left(\alpha_{l} ; v_{2}\left(\mathbf{P}_{l-1}\right)\right)$

- $\mathbf{g}=D_{l}$

$\left(\alpha_{i} ; \mathbf{P}_{i-1} \times \mathbf{Q}_{2(l-i)-2}\right)$ for $1 \leqslant i \leqslant l-2,\left(\alpha_{l-1} ; G r(2, l-2)\right),\left(\alpha_{l} ; G r(2, l-2)\right)$

- $\mathbf{g}=E_{6}$

$\left(\alpha_{1} ; G r^{I I}(5,5)\right),\left(\alpha_{2} ; G r(3,3)\right),\left(\alpha_{3} ; \mathbf{P}_{1} \times G r(2,3)\right),\left(\alpha_{4} ; \mathbf{P}_{1} \times \mathbf{P}_{2} \times \mathbf{P}_{2}\right)$

- $\mathbf{g}=E_{7}$

$$
\begin{aligned}
& \left(\alpha_{1} ; G r^{I I}(6,6)\right),\left(\alpha_{2} ; G r(3,4)\right),\left(\alpha_{3} ; \mathbf{P}_{1} \times G r(2,4)\right),\left(\alpha_{4} ; \mathbf{P}_{1} \times \mathbf{P}_{2} \times \mathbf{P}_{3}\right), \\
& \left(\alpha_{5} ; \mathbf{P}_{2} \times G r(2,3)\right),\left(\alpha_{6} ; \mathbf{P}_{1} \times G r^{I I}(5,5)\right),\left(\alpha_{7} ; \mathbf{E}_{6}\right)
\end{aligned}
$$

- $\mathbf{g}=E_{8}$

$\left(\alpha_{1} ; G r^{I I}(7,7)\right),\left(\alpha_{2} ; G r(3,5)\right),\left(\alpha_{3} ; \mathbf{P}_{1} \times G r(2,5)\right),\left(\alpha_{4} ; \mathbf{P}_{1} \times \mathbf{P}_{2} \times \mathbf{P}_{4}\right)$,

$\left(\alpha_{5} ; \mathbf{P}_{3} \times G r(2,3)\right),\left(\alpha_{6} ; \mathbf{P}_{2} \times G r^{I I}(5,5)\right),\left(\alpha_{7} ; \mathbf{P}_{1} \times \mathbf{E}_{6}\right),\left(\alpha_{8} ; \mathbf{E}_{7}\right)$

- $\mathbf{g}=F_{4}$

$\left(\alpha_{1} ; G r^{I I I}(3,3)\right),\left(\alpha_{2} ; \mathbf{P}_{1} \times v_{2}\left(\mathbf{P}_{2}\right)\right),\left(\alpha_{3} ; \mathbf{P}_{1} \times \mathbf{P}_{2}\right),\left(\alpha_{4} ; G r^{I I}(3,3)\right)$

- $\mathbf{g}=G_{2}$

$\left(\alpha_{1} ; \mathbf{P}_{1}\right),\left(\alpha_{2} ; v_{3}\left(\mathbf{P}_{1}\right)\right)$

In the list, $\mathbf{Q}_{k}$ denotes the $k$-dimensional smooth hyperquadric, $\operatorname{Gr}(k, l)$ denotes the Grassmannian of $k$-dimensional subspaces in $(k+l)$-dimensional vector space, $G r^{I I}(k, k)$ denotes the orthogonal Grassmannian of $k$-dimensional isotropic subspaces in a $2 k$-dimensional orthogonal vector space, $G r^{I I I}(k, k)$ denotes the Lagrangian Grassmannian of a $2 k$-dimensional symplectic vector space, and $\mathbf{E}_{6}$ (resp. $\mathbf{E}_{7}$ ) denotes the Hermitian symmetric space with the group $E_{6}$ (resp. $E_{7}$ ). $v_{2}\left(\mathbf{P}_{k}\right)$ (resp. $v_{3}\left(\mathbf{P}_{k}\right)$ ) denotes the 2nd (resp. 3rd) Veronese embedding of the projective space. Except these Veronese embeddings of projective spaces, all other irreducible Hermitian symmetric spaces are embedded in a minimal way and the product stands for the Segre embedding coming from tensor product of the embeddings of each factor.

Now let $G$ (resp. $P$ ) be a complex Lie group with Lie algebra $\mathrm{g}$ (resp. p). The quotient variety $G / P$ is called the rational homogeneous space associated to the simple root $\alpha_{i}$. The quotient map $G \rightarrow G / P$ defines a $P$-principal bundle on $G / P$. The left action of $P$ on the reductive group $L=P / U$ where $U$ is the unipotent radical of $P$, induces an $L$-principal bundle $\mathbf{L}$ on $G / P$. The Picard group of $G / P$ is generated by an ample line bundle $\mathcal{L}$. This line bundle $\mathcal{L}$ is homogeneous and is associated to $\mathbf{L}$ by a 1-dimensional representation of $L$. This representation can be described as follows. Let $\alpha_{i}$ be the simple root defining $P$. Let $H_{\alpha_{i}} \in \mathbf{h}$ be its coroot. The center of the reductive group $L=P / U$ has Lie algebra $\mathbf{C} H_{\alpha_{i}}$. Hence a $\mathbf{Z}$-functional on $\mathbf{Z} H_{\alpha_{i}}$ induces a character of $L$, giving rise to a homogeneous line bundle on $G / P$. The line bundle $\mathcal{L}$ is the one associated to the functional having value 1 on $H_{\alpha_{i}}$. It is well-known that $\mathcal{L}$ is very ample. 
For example, when $G / P=\mathbf{P}_{1}, \mathbf{g}=\mathbf{s l}_{2}$ has a unique simple root and corresponding coroot. A functional having value $k \in \mathbf{Z}$ on the coroot gives rise to the line bundle $\mathcal{O}(k)$ on $\mathbf{P}_{1}$.

On our rational homogeneous space $G / P$, we have rational curves which are lines under the embedding defined by $\mathcal{L}$. Let $\alpha_{i}$ be the simple root defining $P$ and $H_{\alpha_{i}} \in \mathbf{h}$ be its coroot. Let $\mathbf{s}_{\alpha_{i}} \subset \mathbf{g}$ be the subalgebra isomorphic to $\mathbf{s l}_{2}$ such that $\mathbf{s}_{\alpha_{i}} \cap \mathbf{h}=\mathbf{C} H_{\alpha_{i}}$ and $H_{\alpha_{i}}$ is the coroot for $\mathbf{s}_{\alpha_{i}}$. The orbit of $o \in G / P$ under the subgroup $S_{\alpha_{i}} \subset G$ with Lie algebra $\mathbf{s}_{\alpha_{i}}$ is a rational curve and will be denoted by $C_{\alpha_{i}}$. Note that the character of $L$ defining $\mathcal{L}$ has value 1 on $H_{\alpha_{i}}$. Thus $C_{\alpha_{i}}$ is a line under the embedding of $G / P$ defined by $\mathcal{L}$. Under the natural identification of $\mathbf{g}_{1}$ as a subspace of the tangent space $T_{o}(G / P), H_{\alpha_{i}}$ is a tangent vector of the line $C_{\alpha_{i}}$ at the point $o \in G / P$.

So far all our discussions work for any simple root $\alpha_{i}$. But for the next Proposition we need to assume that $\alpha_{i}$ is a long simple root.

PROPOSITION 1. - If $\alpha_{i}$ is a long simple root of $\mathrm{g}$, then the Chow space of lines through the base point $o \in G / P$ is isomorphic to the highest weight variety $\mathbf{P} W \subset \mathbf{P g}_{1}$.

Proof. - Each point $w \in \mathbf{P} W$ can serve as the highest weight vector $H_{\alpha_{i}}$ under a suitable choice of the Cartan subalgebra $\mathbf{h}$ and the Weyl chamber. Thus we have a line $C_{w}$ whose tangent vector at $o$ is given by $w$. Thus $\mathbf{P} W$ is a subvariety of the Chow space of lines through $o$.

We claim that $\mathbf{P} W$ is an irreducible component of the Chow space. It suffices to show that the dimension of the deformation of a line fixing a point on $G / P$ cannot exceed the dimension of $\mathbf{P} W$. The former is bounded by $h^{0}\left(C_{w}, N \otimes \mathcal{O}(-1)\right)$ where $N$ is the normal bundle of the line in $G / P$. Since the normal bundle is semi-positive, $h^{0}\left(C_{w}, N \otimes \mathcal{O}(-1)\right)=C_{w} \cdot K_{G / P}^{-1}-2$. To calculate the anti-canonical degree of $C_{w}$, we use Grothendieck's splitting theorem for principal bundles on $\mathbf{P}_{1}$ with reductive structure groups and associated vector bundles [2].

THEOREM (Grothendieck). - Let $\mathcal{O}(1)^{*}$ be the $\mathbf{C}^{*}$-principal bundle on $\mathbf{P}_{1}$ corresponding to the line bundle $\mathcal{O}(1)$. Let L be a reductive complex Lie group. Up to conjugation, any L-principal bundle on $\mathbf{P}_{1}$ is associated to $\mathcal{O}(1)^{*}$ by a group homomorphism from $\mathbf{C}^{*}$ to a maximal torus of $L$. If $H$ is the coroot of $\mathbf{s l}_{2}$, such a group homomorphism is determined by the image of $H$ in $\mathbf{h}$, a fixed Cartan subalgebra of $L$. Given a representation of $L$ with weights $\mu_{1}, \ldots, \mu_{l} \in \mathbf{h}^{*}$, the associated vector bundle on $\mathbf{P}_{1}$ splits as $\mathcal{O}\left(\mu_{1}(H)\right) \oplus \cdots \oplus \mathcal{O}\left(\mu_{l}(H)\right)$, where $\mu_{j}(H)$ denotes the value of $\mu_{j}$ on the image of $H$ in $\mathbf{h}$.

Note that $T_{o}(G / P)$ can be naturally identified with $\mathbf{g} / \mathbf{p}$. So the Chern number of $T(G / P)$ is equal to the sum of Chern numbers of the vector bundles associated to the $L$-principal bundle $\mathbf{L}$ via the representations of $L$ on $\mathbf{g}_{1}, \ldots, \mathbf{g}_{m}$. Hence by Grothendieck's theorem, the Chern number of $T(G / P)$ restricted to $C_{\alpha_{i}}$ is $\sum_{\beta \in \Phi_{1} \cup \cdots \cup \Phi_{m}} \beta\left(H_{\alpha_{i}}\right)$. Since $\alpha_{i}$ is a long root,

$$
\beta\left(H_{\alpha_{i}}\right)= \begin{cases}2 & \text { if } \beta=\alpha_{i}, \\ 1 & \text { if } \beta \neq \alpha_{i} \text { and } \beta-\alpha_{i} \in \Phi, \\ -1 & \text { if } \beta \neq \alpha_{i} \text { and } \beta+\alpha_{i} \in \Phi\end{cases}
$$

From $\alpha_{i} \in \Phi_{1}$, the Chern number is

$$
\begin{aligned}
\sum_{\beta \in \Phi_{1} \cup \cdots \cup \Phi_{m}} \beta\left(H_{\alpha_{i}}\right)= & 2+\sharp\left\{\beta \in \Phi_{1} \cup \cdots \cup \Phi_{m}, \beta \neq \alpha_{i}, \beta-\alpha_{i} \in \Phi\right\} \\
& -\sharp\left\{\beta \in \Phi_{1} \cup \cdots \cup \Phi_{m}, \beta \neq \alpha_{i}, \beta+\alpha \in \Phi\right\} \\
= & 2+\sharp\left\{\beta \in \Phi_{1}, \beta-\alpha_{i} \in \Phi_{0}\right\} \\
= & 2+\sharp\left\{\gamma \in \Phi_{0}, \alpha+\gamma \in \Phi_{0}\right\} \\
= & 2+\operatorname{dim}\left(\left[\mathrm{g}_{0}, H_{\alpha_{i}}\right]\right) .
\end{aligned}
$$


It follows that $h^{0}\left(C_{w}, N \otimes \mathcal{O}(-1)\right)=\operatorname{dim}\left(\left[1, H_{\alpha_{i}}\right]\right)$. But $\operatorname{dim}\left(\left[1, H_{\alpha_{i}}\right]\right)$ is exactly the dimension of $\mathbf{P} W$. This proves that $\mathbf{P} W$ is an irreducible component of the Chow space of lines through $o$.

It remains to show that the Chow space of lines through $o$ is irreducible. A line is determined by its tangent vector at $o$. Thus if there exists a line different from $C_{w}$, its tangent vector will be contained in $\mathbf{T}_{o}(G / P)-\mathbf{P} W$. From Proposition 5.2 in [6], the closure of the $P$-orbit of such a vector intersects $\mathbf{P} W$. Since the limit of a family of lines is again a line, this implies that the component $\mathbf{P} W$ is not smooth. However $\mathbf{P} W$ is homogeneous and $h^{1}\left(C_{w}, N \otimes \mathcal{O}(-1)\right)=0$ since $N$ is semi-positive, so the Chow component $\mathbf{P} W$ is smooth, a contradiction.

Remark 1. - As complex manifolds, the rational homogeneous space associated to $\alpha_{l}$ for $\mathbf{g}=B_{l}$ is biholomorphic to that associated to $\alpha_{l}$ for $\mathbf{g}=D_{l+1}$. Also the rational homogeneous space associated to $\alpha_{1}$ for $\mathbf{g}=G_{2}$ is isomorphic to $\mathbf{Q}_{5}$ which is associated to $\alpha_{1}$ for $\mathbf{g}=B_{3}$. Thus when we study complex structure of $G / P$, these two cases can be regarded as rational homogeneous spaces associated to long simple roots.

Remark 2. - When $\alpha_{i}$ is a short simple root, Proposition 1 does not hold. The Chow space of lines through $o$ contains, but is strictly bigger than, $\mathbf{P} W$. It is not contained in $\mathbf{P g}_{1}$ and excepting the cases mentioned in Remark 1, it is not homogeneous.

\section{Rigidity of the normalized Chow spaces}

Let us recall some basic facts from deformation theory of rational curves (cf. Section 2 of [5] or [11]). Let $X$ be a Fano manifold of Picard number 1 and $x \in X$ be a generic point. Let $\mathcal{K}_{x}$ be an irreducible component of the normalized Chow space of rational curves of minimal degree through $x$. Then $\mathcal{K}_{x}$ is a smooth projective variety. If the anti-canonical degree of members of $\mathcal{K}_{x}$ is $p+2$, then $\mathcal{K}_{x}$ has dimension $p$, and for a generic member $C$ of $\mathcal{K}_{x}$,

$$
\left.T(X)\right|_{C}=\mathcal{O}(2) \oplus[\mathcal{O}(1)]^{p} \oplus \mathcal{O}^{n-1-p} .
$$

Define the tangent map $\tau_{x}: \mathcal{K}_{x} \rightarrow \mathbf{P} T_{x}(X)$ by assigning the tangent vector at $x$ to each member of $\mathcal{K}_{x}$ which is smooth at $x$. This is a generically finite rational map and its strict image is denoted by $\mathcal{C}_{x}$, called the variety of minimal rational tangents at $x$. Suppose $X$ is embedded in some projective space $\mathbf{P}_{N}$ and a minimal rational curve through a generic point $x$ is a line in $\mathbf{P}_{N}$. Since lines through $x$ in $\mathbf{P}_{N}$ are determined by their tangent vectors at $x, \tau_{x}$ is an embedding. This is the case for our $G / P$. In particular, when $P$ is associated to a long simple root, Proposition 1 implies that $\mathcal{K}_{o} \cong \mathcal{C}_{o} \cong \mathbf{P} W$ and $\tau_{o}$ is an embedding described in the list of highest weight varieties in Section 1.

We now go to the situation of the Main Theorem. Let $\pi: \mathcal{X} \rightarrow \Delta$ be a smooth projective morphism from a complex manifold to the unit disc. Suppose the fiber $X_{t}:=\pi^{-1}(t)$ is biholomorphic to $G / P$ associated to a long simple root for each $t \neq 0$. Let us use the same symbol $\mathcal{L}$ to denote the line bundle on $\mathcal{X}$ whose restriction to $X_{t}$ is equivalent to the line bundle $\mathcal{L}$ on $G / P$. Choose a generic point $x \in X_{0}$ and a section $\sigma: \Delta \rightarrow \mathcal{X}$ of $\pi$ satisfying $\pi(0)=x$. Let $\rho: \mathcal{K}_{\sigma} \rightarrow \Delta$ be the family of normalized Chow spaces $\mathcal{K}_{\sigma(t)}$ of minimal rational curves through $\sigma(t)$ in $X_{t}$. Then $\rho$ is a smooth projective morphism by the same proof as Proposition 4 in [5] or Proposition 8 in [3]. The goal of this section is to prove the following.

PROPOSITION 2. - The family $\rho: \mathcal{K}_{\sigma} \rightarrow \Delta$ is a trivial family, namely, its fiber at $t=0$ is also isomorphic to $\mathbf{P} W$.

Proof. - From the list of highest weight varieties in Section 1, we see that $\mathbf{P} W$ belongs to (at least) one of the following.

$4^{\mathrm{e}}$ SÉRIE - TOME $35-2002-\mathrm{N}^{\circ} 2$ 
(i) $\mathbf{P} W$ is an irreducible Hermitian symmetric space.

(ii) $\mathbf{P} W$ is the product of two projective spaces.

(iii) $\mathbf{P} W \cong S_{1} \times S_{2}$ where $S_{1} \cong \mathbf{P}_{k}$ and $S_{2}$ is a hyperquadric.

(iv) $\mathbf{P} W \cong S_{1} \times S_{2}$ where $S_{1} \cong \mathbf{P}_{k}$ and $S_{2}$ is a Hermitian symmetric space of rank 2 with $\operatorname{dim}\left(S_{2}\right)>k:=\operatorname{dim}\left(S_{1}\right)$.

For the case (i), Proposition 2 follows from the result of [5]. For the case (ii), Proposition 3 was proved in Section 3 of [5]. Thus we will only consider the cases (iii) or (iv). In these cases, either $S_{2}$ is irreducible or the product of two projective spaces. Let $\zeta_{1}$ be the hyperplane line bundle on $S_{1} \cong \mathbf{P}_{k}$ and $\zeta_{2}$ be the ample line bundle on $S_{2}$ which is the generator of $\operatorname{Pic}\left(S_{2}\right)$ if $S_{2}$ is irreducible and is the tensor product of hyperplane bundles of each factor when $S_{2}$ is the product of two projective spaces. Let $\zeta=\zeta_{1} \otimes \zeta_{2}$. We say that a curve on $\mathcal{C}_{o}$ is a line (resp. a conic), if it has degree 1 (resp. 2) with respect to $\zeta$. Let $\xi$ (resp. $\xi_{1}$, resp. $\xi_{2}$ ) be the line bundle on $\mathcal{K}_{\sigma}$ so that its restriction to $\rho^{-1}(t)$ is $\zeta$ (resp. $\zeta_{1}$, resp. $\zeta_{2}$ ) for $t \neq 0$.

LEMma 1. - Let $l_{t} \subset \mathcal{K}_{\sigma(t)}$ be a family of curves so that $l_{t}$ is a line on $S_{1} \times S_{2} \cong \mathcal{K}_{\sigma(t)}$ for all $t \neq 0$. Then $l_{0}$ is irreducible and reduced as a cycle in $\mathcal{K}_{\sigma(0)}$.

Proof. - For $t \neq 0$, a line in $\mathcal{K}_{\sigma(t)}$ corresponds to a family of lines in $G / P$ passing through a fixed point $o \in G / P$, which span a surface of degree 1 with respect to $\mathcal{L}$. Given a family of rational curves $l_{t} \subset \mathcal{K}_{\sigma(t)}$ of degree 1 with respect to $\xi$, we have a corresponding family of surfaces $R_{t} \subset \mathcal{X}_{t}$ of degree 1 with respect to $\mathcal{L}$. Since $\mathcal{L}$ is ample on $\mathcal{X}_{0}$, the limit $R_{0}$ must be a reduced irreducible surface. It follows that the limit $l_{0}$ is a reduced irreducible rational curve on $\mathcal{K}_{\sigma(0)}$.

Note that for any polarized projective manifold $X$ and an integer $N$, there exists a non-empty Zariski open subset $X^{*} \subset X$ with the property that for any irreducible rational curve $C$ of degree $\leqslant N$ with respect to the given polarization, $\left.T(X)\right|_{C}$ is semipositive if $C$ contains a point of $X^{*}$ (e.g. the argument of [11, II.3.11]).

LEMMA 2. - Let $y \in \mathcal{K}_{\sigma(0)}$ be a generic point. Let $c_{t} \subset \mathcal{K}_{\sigma(t)}$ be a family of curves so that $c_{t}$ is a conic on $S_{1} \times S_{2} \cong \mathcal{K}_{\sigma(t)}$ for all $t \neq 0$ and $c_{0}$ contains $y$. Then $c_{0}$ is either irreducible or has two components of degree 1 with respect to $\xi$.

Proof. - A conic on $S_{1} \times S_{2}$ can be degenerated to a union of two lines. Thus for $t \neq 0$, a conic on $\mathcal{K}_{\sigma(t)}$ corresponds to a surface of degree 2 in $X_{t}$ with respect to $\mathcal{L}$. By the same argument as in Lemma $1, c_{0}$ can have at most two components. Suppose it has two components $c_{00}$ and $c_{01}$. One of them, say $c_{00}$, contains $y$ and we may assume $\left.T\left(\mathcal{K}_{\sigma(0)}\right)\right|_{c_{00}}$ is semipositive from the genericity of $y$. From $H^{1}\left(c_{00}, T\left(\mathcal{K}_{\sigma(0)}\right)\right)=0$ and Kodaira's stability [10], we have a family of rational curves $C_{t}^{\prime} \subset \mathcal{K}_{\sigma(t)}$ so that $C_{0}^{\prime}=c_{00}$. In particular, $c_{00}$ has positive degree with respect to $\xi$. Suppose that $c_{00}$ has degree $>1$ with respect to $\xi$. Then the surface in $X_{t}$ corresponding to $C_{t}^{\prime}$ is of degree $>1$ with respect to $\mathcal{L}$. It follows that the surface in $X_{0}$ corresponding to $c_{00}$ has degree $>1$ with respect to $\mathcal{L}$. This is not possible because the total degree of the surfaces corresponding to $c_{00} \cup c_{01}$ is 2 . Hence $c_{00}$ has degree 1 with respect to $\xi$ and so does $c_{01}$.

We have two foliations $\mathcal{E}$ and $\mathcal{F}$ on $\mathcal{K}_{\sigma}$ so that the leaves of $\left.\mathcal{E}\right|_{\mathcal{K}_{\sigma(t)}}, t \neq 0$ (resp. $\left.\mathcal{F}\right|_{\mathcal{K}_{\sigma(t)}}$ ) are the $S_{1}$-factors (resp. $S_{2}$-factors ) of $\mathcal{K}_{\sigma(t)} \cong S_{1} \times S_{2}$. They define meromorphic foliations on $\mathcal{K}_{\sigma(0)}$.

Lemma 3. - Let $y \in \mathcal{K}_{\sigma(0)}$ be a generic point and $\mu: \Delta \rightarrow \mathcal{K}_{\sigma}$ be a section of $\rho$ with $\mu(0)=y$. Let $P_{t}$ be the $\mathcal{E}$-leaf and $Q_{t}$ be the $\mathcal{F}$-leaf through $\mu(t)$ on $\mathcal{K}_{\sigma(t)}, t \neq 0$. Then the limits $P_{0}$ and $Q_{0}$ are irreducible and reduced as cycles in $\mathcal{K}_{\sigma(0)}$. 
Proof. - Since $P_{t}$ and $Q_{t}$ have intersection number 1 for all $t \in \Delta$, the reducedness of $P_{0}$ and $Q_{0}$ is immediate if they are irreducible.

Suppose $P_{0}$ is reducible. We can choose two families of distinct points $\alpha_{t}, \beta_{t} \in P_{t}$ so that $\alpha_{0}$ and $\beta_{0}$ lie on different components of $P_{0}$. Since $P_{t} \cong \mathbf{P}_{k}$ for $t \neq 0$, there exists a line $l_{t} \subset P_{t}$ joining $\alpha_{t}$ and $\beta_{t}$. By Lemma 1 , the limit $l_{0}$ must be irreducible while $\alpha_{0}, \beta_{0} \in l_{0}$, a contradiction. Thus $P_{0}$ is irreducible.

To prove the irreducibility of $Q_{0}$, we consider the case (iii) and the case (iv) separately.

For the case (iii), we will use the following property of the hyperquadric $S_{2}$ : given two generic points $A, B \in S_{2}$, the union of all conics passing through $A$ and $B$ covers $S_{2}$. This is because the tangent bundle of the hyperquadric splits as a direct sum of $\mathcal{O}(2)$ 's over a conic. Suppose $Q_{0}$ is reducible. Choose two generic points $A_{0}, B_{0}$ in one of the component of $Q_{0}$ so that both $A_{0}$ and $B_{0}$ are very general. Choose two families of points $A_{t}, B_{t} \in Q_{t}$ converging to $A_{0}$ and $B_{0}$. Consider the union of all conics through $A_{t}$ and $B_{t}$. By the above mentioned property of $S_{2}$, the limits of these conics must cover $Q_{0}$. Since $Q_{0}$ is reducible, this means that for any family of conics $c_{t}$ passing through $A_{t}$ and $B_{t}$ its limit is reducible and one of the components is a line passing through $A_{0}$ and $B_{0}$. The union of such lines must cover one component of $Q_{0}$. By Mori's bend-and-break [11, II.5], this family of lines through $A_{0}$ and $B_{0}$ must degenerate to a union of two rational curves. But this gives a contradiction to the degree of corresponding surface in $X_{0}$ as in the proofs of Lemmas 1 and 2.

For the case (iv), we will use the following property of Hermitian symmetric space $S_{2}$ of rank 2: conics through a given point on $S_{2}$ cover $S_{2}$. This is a consequence of the polydisc theorem (Ch. 5 (1.1) in [12]). If $Q_{0}$ is reducible, choose $A_{t}, B_{t} \in Q_{t}$ so that $A_{0}$ and $B_{0}$ are generic points of distinct components of $Q_{0}$. We may assume that $A_{0}$ is a very general point. We can find a family of conics $c_{t} \subset Q_{t}$ containing $A_{t}$ and $B_{t}$. The limit $c_{0}$ cannot be irreducible, and must be the union of two irreducible curves of degree 1 with respect to $\xi$ by Lemma 2 . Fixing $A_{0}$ and varying $B_{0}$, we get irreducible rational curves of degree 1 through $A_{0}$ which cover a component of $Q_{0}$. Since $A_{0}$ is very general, we may assume that these degree 1 curves through $A_{0}$ are limits of families of degree 1 curves through $A_{t}$ in $\mathcal{K}_{\sigma(t)}$ by Kodaira's stability [10] as in the proof of Lemma 2 . Thus on $\mathcal{K}_{\sigma(t)}$, we get a $\left(\operatorname{dim}\left(S_{2}\right)-1\right)$-dimensional family of lines through a fixed point, but this is impossible because $S_{2}$ is not a projective space and $k<\operatorname{dim}\left(S_{2}\right)$.

Lemma 4. - For a generic point $y \in \mathcal{K}_{\sigma(0)}$, the $\mathcal{E}$-leaf $P$ through $y$ and the $\mathcal{F}$-leaf $Q$ through $y$ intersects transversally at $y$.

Proof. - Suppose not. From the genericity of $y$, there exists a positive dimensional component $R$ of $P \cap Q$ through $y$. Let $P_{t}$ (resp. $Q_{t}$ ) be a family of leaves of $\mathcal{E}$ (resp. $\mathcal{F}$ ) with $P_{0}=P$ (resp. $Q_{0}=Q$ ). Choose two distinct points on $R$ generically. Then there exists a family of lines $l_{t}$ on $P_{t}$ so that $l_{0}$ contains these two points on $R$. We can choose a section of $\xi_{1}$ whose zero section $H$ is a hypersurface consisting of $\mathcal{F}$-leaves so that $Q \subset H$. Since $l_{0}$ has degree 1 with respect to $\xi_{1}$ and contains at least two points of $H$, we see that $l_{0} \subset H$. This implies that $l_{0} \subset Q$. From the genericity of $y$, we can assume that $l_{0}$ passes through a generic point of $Q$. We know that $\xi_{2}$ is big on $Q$ because it is ample on $Q_{t}$. On the other hand, $l_{0} \cdot \xi_{2}=0$, a contradiction.

We are ready to finish the proof of Proposition 2. From above, $\mathcal{E}$ and $\mathcal{F}$ define two transversal foliations at generic points of $\mathcal{K}_{\sigma(0)}$. So we get a direct sum decomposition of the relative tangent bundle of $\rho$ outside a codimension 2 set in $\mathcal{K}_{\sigma}$. Then it extends to a direct sum decomposition everywhere on $\mathcal{K}_{\sigma}$, because the set of all possible direct sum decompositions of a given vector space is an affine variety. It follows that the foliations $\mathcal{E}$ and $\mathcal{F}$ on $\mathcal{K}_{\sigma}$ have no singularity. Since $\mathcal{K}_{\sigma(0)}$ is simply connected, $\mathcal{K}_{\sigma(0)}$ is biholomorphic to the product of smooth deformations of $S_{1}$ 
and $S_{2}$. This finishes the proof when $S_{2}$ is irreducible by the result of [5]. When $S_{2}$ is the product of two projective spaces, we apply the same argument as above to the family of leaves $Q_{t}$, as was done in Section 3 of [5], to conclude.

\section{Symbol algebra of the differential system}

Let us recall some definitions in the theory of differential systems [16]. Given a distribution $D$ on a complex manifold $X$, define the weak derived system $D^{k}$ inductively by

$$
\begin{aligned}
& D^{1}=D, \\
& D^{k}=D^{k-1}+\left[D, D^{k-1}\right] .
\end{aligned}
$$

For a generic point $x \in X$ in a neighborhood of which $D^{k}$,s are subbundles of $T(X)$, we define the symbol algebra of $D$ at $x$ as the graded nilpotent Lie algebra $D_{x}^{1}+D_{x}^{2} / D_{x}^{1}+\cdots+D_{x}^{l} / D_{x}^{r-1}$ where $r$ is chosen so that $D^{r+1}=D^{r}$.

When $X$ is a Fano manifold of Picard number 1, choose a component $\mathcal{K}$ of the Chow spaces of rational curves of minimal degree covering $X$. For each generic $x \in X$, let $\mathcal{K}_{x}$ be the subscheme consisting of curves passing through $x$ and $\mathcal{C}_{x} \subset \mathbf{P} T_{x}(X)$ be the variety of minimal rational tangents. Let $\mathcal{V}_{x} \subset T_{x}(X)$ be the linear span of $\mathcal{C}_{x}$ and $\mathcal{V}$ be the meromorphic distribution defined by $\mathcal{V}_{x}$ 's. As an example, consider our $G / P$ associated to a long simple root. We have the $L$-principal bundle $\mathbf{L}$ on $G / P$ induced by the $P$-principal bundle $G \rightarrow G / P$. The $L$-module $\mathbf{g}_{1}$ induces a vector bundle $\mathcal{D}$ on $G / P$. By definition, since $\mathcal{C}_{o}$ is nondegenerate in $\mathbf{g}_{1}$, the distribution $\mathcal{V}$ for $G / P$ agrees with $\mathcal{D}$. Moreover, it is easy to see that the symbol algebra of $\mathcal{D}$ is isomorphic to $\mathbf{g}_{1}+\cdots+\mathbf{g}_{m}$.

Remark 3. - As mentioned in Remark 2, if $G / P$ is associated to a short simple root, the distribution $\mathcal{V}$ is strictly bigger than the distribution defined by $\mathbf{g}_{1}$. For example, $\mathcal{V}$ is the full tangent bundle $T(G / P)$ when $G$ is of type $C$ (symplectic group) and $P$ is associated to a short simple root.

For any Fano manifold $X$ of Picard number 1 and for any choice of $\mathcal{K}$, the distribution $\mathcal{V}$ has the following two properties.

Proposition 3. - Let $[]:, \Lambda^{2} \mathcal{V}_{x} \rightarrow T_{x}(X) / \mathcal{V}_{x}$ be the Frobenius bracket tensor at a generic point $x \in X$. Then for a generic smooth point $v \in \mathcal{C}_{x}$ and $v^{\prime}$ in the tangent space of $\mathcal{C}_{x}$ at $v$, $\left[v, v^{\prime}\right]=0$ when $v$ and $v^{\prime}$ are regarded as vectors in $\mathcal{V}_{x}$.

Proof. - This is just a restatement of Proposition 10 of [5]. Section 4 of [5] was presented under the assumption that $\mathcal{C}_{x}$ is irreducible, but the proof of Proposition $10 \mathrm{did}$ not use this assumption.

Proposition 4. - At a generic point $x \in X$, the symbol algebra of $\mathcal{V}$ has dimension $n=\operatorname{dim}(X)$.

Proof. - By definition, the symbol algebra has dimension $\leqslant n$. If it is strictly less than $n, \mathcal{V}$ is contained in an integrable distribution. This is a contradiction to the assumption that $\mathrm{X}$ is of Picard number 1 by Proposition 2 in [4].

Now let us go to the situation of the Main Theorem. Let $\mathcal{K}$ be a component of the Chow space of $X_{0}$ parametrizing rational curves covering $X_{0}$ which are limits of lines on $X_{t}, t \neq 0$. Let $\tau_{x}: \mathcal{K}_{x} \rightarrow \mathcal{C}_{x} \subset \mathbf{P} T_{x}(X)$ be the tangent map at a generic $x \in X_{0}$. Let $\mathcal{V}_{x} \subset T_{x}\left(X_{0}\right)$ be the linear span of $\mathcal{C}_{x}$ and $\mathcal{V}$ be the meromorphic distribution defined by $\mathcal{V}_{x}$ 's. 
Proposition 5. - At a generic point $x \in X_{0}$, the symbol algebra of $\mathcal{V}$ is isomorphic to $\mathbf{g}_{1}+\cdots+\mathbf{g}_{m}$ as graded nilpotent Lie algebras.

To prove Proposition 5, we need a characterization of the graded nilpotent Lie algebra $\mathbf{g}_{1}+\cdots+\mathbf{g}_{m}$. We need the following lemma which follows immediately from the proof of Serre's Theorem in $[8,18.3]$, using the fact that the subalgebra generated by $\left\{x_{i}, 1 \leqslant i \leqslant l\right\}$ in the Lie algebra $L_{o}$ constructed there is free (see also [14, pp. 48-49]). The latter fact is proved in $[1$, Ch. 8, 4.2] or [9, Theorem 1.2(b)].

LEMMA 5. - Let $\left\{\alpha_{1}, \ldots, \alpha_{l}\right\}$ be a set of simple roots for $\mathrm{g}$ and $\left\langle\alpha_{i}, \alpha_{j}\right\rangle$ be the entries of the Cartan matrix. Let $\left\{x_{i}, y_{i}, h_{i} \mid 1 \leqslant i \leqslant l\right\}$ be the generators of the Serre presentation of $\mathbf{g}$ as given in $[8,18.1]$. Then the subalgebra of $\mathbf{g}$ generated by $\left\{x_{1}, \ldots, x_{l}\right\}$ is the quotient of the free Lie algebra generated by $\left\{x_{1}, \ldots, x_{l}\right\}$ by the relations

$$
\left(a d x_{i}\right)^{-\left\langle\alpha_{j}, \alpha_{i}\right\rangle+1}\left(x_{j}\right)=0
$$

for $i \neq j$.

Using Lemma 5, we get the following characterization of the graded Lie algebra $\mathbf{g}_{0}+\cdots+\mathbf{g}_{m}$.

PROposition 6. - Let $\mathbf{n}=\sum_{i=0}^{\infty} \mathbf{n}_{i}$ be a graded Lie algebra generated by $\mathbf{n}_{0}$ and $\mathbf{n}_{1}$ so that $\mathbf{n}_{0}=\mathbf{g}_{0}$ and $\mathbf{n}_{1}$ is isomorphic to $\mathbf{g}_{1}$ as a $\mathbf{g}_{0}$-module. Let $W \subset \mathbf{n}_{1}$ be the highest weight cone for the representation of $\mathbf{g}_{0}$ on $\mathbf{n}_{1}$. Assume that for any vector $v \in W$, the Lie bracket of $\mathbf{n}$ satisfies $\left[v,\left[\mathbf{g}_{0}, v\right]\right]=0$. Then $\mathbf{n}$ is a quotient of the graded Lie algebra $\mathbf{g}_{0}+\cdots+\mathbf{g}_{m}$.

Proof. - Let $\mathbf{m} \subset \mathbf{g}_{0}$ be the subalgebra generated by $\left\{x_{i}, i \neq k\right\}$ where $\alpha_{k}$ is the long simple root defining $\mathbf{p}$. Consider the subalgebra $\mathbf{n}^{\prime}=\mathbf{m}+\mathbf{n}_{1}+\cdots$ of $\mathbf{n}$. As an abstract Lie algebra, $\mathbf{n}^{\prime}$ is generated by $\left\{x_{i}, 1 \leqslant i \leqslant l\right\}$. It satisfies all the relations

$$
\left(a d x_{i}\right)^{-\left\langle\alpha_{j}, \alpha_{i}\right\rangle+1}\left(x_{j}\right)=0
$$

for $i \neq j$. In fact, if $j \neq k$ and $i \neq k$, this relation is just one of the Serre relations for $\mathbf{g}_{0}$. If $j=k$, this relation concerns the action of $\mathbf{m}$ on $\mathbf{n}_{1}$, which we assumed to be equivalent to the action of $\mathbf{m}$ on $\mathbf{g}_{1}$ for which the relation is just one of the Serre relations. When $i=k$ and $\left\langle\alpha_{j}, \alpha_{k}\right\rangle=0$, this follows again from the action of $\mathbf{m}$ on $\mathbf{n}_{1}$. Since $\alpha_{k}$ is a long root, the only remaining case is when $i=k$ and $\left\langle\alpha_{j}, \alpha_{k}\right\rangle=-1$, for which the relation is just

$$
\left[x_{k},\left[x_{k}, x_{j}\right]\right]=0 .
$$

But this is satisfied from the assumption that $\left[v,\left[\mathbf{g}_{0}, v\right]\right]=0$ for any $v \in W$. It follows from Lemma 5 that $\mathbf{n}^{\prime}$ is a quotient of the subalgebra of $\mathbf{g}$ generated by $\left\{x_{1}, \ldots, x_{l}\right\}$, which implies Proposition 6.

Now we have the following characterization of the graded Lie algebra $\mathbf{g}_{1}+\cdots+\mathbf{g}_{m}$.

Proposition 7. - Let $W \subset \mathbf{g}_{1}$ be the cone of highest weight vectors as a $\mathbf{g}_{0}$-module and $\mathbf{F}\left(\mathbf{g}_{1}\right)$ be the graded free Lie algebra generated by $\mathbf{g}_{1}$. We consider the ideal I of $\mathbf{F}\left(\mathbf{g}_{1}\right)$ generated by the relations $\left[v,\left[\mathbf{g}_{0}, v\right]\right]=0$ for all $v \in W$. Let us denote the quotient graded algebra $\mathbf{F}\left(\mathbf{g}_{1}\right) /$ I by $\mathbf{n}_{1}+\mathbf{n}_{2}+\cdots$. Then $\mathbf{n}_{1}+\mathbf{n}_{2}+\cdots$ is isomorphic to the nilpotent graded Lie algebra $\mathbf{g}_{1}+\cdots+\mathbf{g}_{m}$.

Proof. $-\mathbf{g}_{0}$-action on $\mathbf{g}_{1}$ induces a $\mathbf{g}_{0}$-action on the tensor algebra of $\mathbf{g}_{1}$ as a derivation, making $\mathbf{g}_{0}+\mathbf{F}\left(\mathbf{g}_{1}\right)$ into a graded Lie algebra whose 0 -degree part is exactly $\mathbf{g}_{0}$. Since the ideal $I$ 
is invariant under the action of $\mathbf{g}_{0}, \mathbf{g}_{0}+\mathbf{n}_{1}+\mathbf{n}_{2}+\cdots$ becomes a graded Lie algebra. Setting $\mathbf{n}_{0}=\mathbf{g}_{0}$, we can apply Proposition 6 to identify $\mathbf{n}_{1}+\mathbf{n}_{2}+\cdots$ with $\mathbf{g}_{1}+\cdots+\mathbf{g}_{m}$.

Now we are ready to finish the proof of Proposition 5.

Proof of Proposition 5. - Choose a section $\sigma: \Delta \rightarrow \mathcal{X}$ of $\pi: \mathcal{X} \rightarrow \Delta$ so that $x=\sigma(0)$ is a generic point of $X_{0}=\pi^{-1}(0)$. The family $\mathcal{K}_{\sigma}$ of normalized Chow spaces of minimal rational curves through $\sigma$ is a trivial family of $\mathbf{P} W$ by Proposition 2. For $t \neq 0$, the tangent map $\tau_{\sigma(t)}: \mathcal{K}_{\sigma(t)} \rightarrow \mathbf{P} T_{\sigma(t)}\left(X_{t}\right)$ is an embedding into $\mathbf{P} \mathcal{D}_{\sigma(t)}=\mathbf{P g}_{1}$ given by a complete linear system of the line bundle $\xi$ on $\mathcal{K}_{\sigma}$ defined in Section 2. Thus $\tau_{\sigma(0)}$ is a rational map defined by a subsystem of this complete linear system. Namely, $\tau_{\sigma(0)}$ is induced by a projection $\mathbf{g}_{1} \rightarrow \mathcal{V}_{x}$. Let $W^{\prime} \subset \mathcal{V}_{x}$ be the image of the highest weight cone $W \subset \mathbf{g}_{1}$ under the projection. Then $\mathbf{P} W^{\prime}=\mathcal{C}_{x}$, the variety of minimal rational tangents at $x$.

Consider the free lie algebra $\mathbf{F}\left(\mathcal{V}_{x}\right)$ generated by $\mathcal{V}_{x}$ and let $J$ be its ideal generated by the relations given by $\left[v, v^{\prime}\right]$ where $v$ is a smooth point of $W^{\prime}$ and $v^{\prime}$ is a vector in the tangent space of the cone $W^{\prime}$ at $v$. Then the quotient graded Lie algebra $\mathbf{F}\left(\mathcal{V}_{x}\right) / J$ is a quotient of $\mathbf{g}_{1}+\cdots+\mathbf{g}_{m}$ by Proposition 7 because $J$ contains the image of $I$ under the natural graded Lie algebra homomorphism $\mathbf{F}\left(\mathbf{n}_{1}\right) \rightarrow \mathbf{F}\left(\mathcal{V}_{x}\right)$. From Proposition 3, the symbol algebra of $\mathcal{V}$ at $x$ is a quotient algebra of $\mathbf{F}\left(\mathcal{V}_{x}\right) / J$, thus a quotient algebra of $\mathbf{g}_{1}+\cdots+\mathbf{g}_{m}$. If the symbol algebra is not isomorphic to $\mathbf{g}_{1}+\cdots+\mathbf{g}_{m}$, it has dimension strictly smaller than $n=\operatorname{dim}(G / P)$, a contradiction to Proposition 4.

Our Main Theorem follows from Proposition 5 via the works of Tanaka and Yamaguchi ([15] and p. 479 of [16]. See also 3.10 of [13] for a more general treatment). Let us briefly summarize their works. Let $G / P$ be a rational homogeneous space associated to a simple root. Assume that $G / P$ is not a symmetric space or a homogeneous contact manifold. Given a differential system $D$ on a complex manifold whose symbol algebra at a generic point is isomorphic to $\mathbf{g}_{1}+\cdots+\mathbf{g}_{m}$, there exists a natural holomorphic $P$-principal bundle $\mathcal{P}$ over an open neighborhood $\mathcal{U}$ of a generic point with a canonical choice of g-valued 1-form $\omega$, called the Cartan connection, so that if the Maurer-Cartan equation $d \omega+\frac{1}{2}[\omega, \omega]=0$ holds, then there exists a biholomorphic map of $\mathcal{U}$ to an open subset of $G / P$ which sends the distribution $D$ to the distribution $\mathcal{D}$ on $G / P$ induced by $\mathbf{g}_{1}$. The construction of $\omega$ given in [15] or 3.10 of [13] can be carried out when we are given a family of complex manifolds with a family of differential systems whose symbol algebras are isomorphic to $\mathbf{g}_{1}+\cdots+\mathbf{g}_{m}$.

Proof of Main Theorem. - From [5] and [3], we may assume that $G / P$ is not a symmetric space or a homogeneous contact manifold. By Proposition 5, we are given a family of meromorphic distributions $\mathcal{V}_{t}$ on $\mathcal{X}$ whose symbol algebra at a generic point of $X_{t}$ is $\mathbf{g}_{1}+\cdots+\mathbf{g}_{m}$ for all $t \in \Delta$. We can apply the construction of [15] or 3.10 of [13] to a family of neighborhoods $\mathcal{U}_{t}$ of $x \in X_{0}$ to get a $P$-principal bundle $\mathcal{P}$ over $\mathcal{U}:=\bigcup_{t \in \Delta} \mathcal{U}_{t}$ with the Cartan connection $\omega$ on $\mathcal{P}$. Since the Maurer-Cartan equation holds for $t \neq 0$, it holds also for $t=0$. Thus there exists a biholomorphic map from $\mathcal{U}_{0}$ to an open subset of $G / P$ sending $\mathcal{V}$ to $\mathcal{D}$. From the upper-semicontinuity of $h^{0}\left(X_{t}, T\left(X_{t}\right)\right)$, the Lie algebra aut $\left(X_{0}\right)$ of infinitesimal automorphisms of $X_{0}$ has $\operatorname{dimension} \geqslant \operatorname{dim}(\mathrm{g})$. By Corollary 5.4 of [16], the Lie algebra of infinitesimal automorphisms of $\mathcal{U}_{0}$ preserving $\mathcal{V}_{0}$ is isomorphic to $\mathrm{g}$. Thus $\operatorname{aut}\left(X_{0}\right) \cong \mathrm{g}$ and the isomorphism is induced by the biholomorphism from $\mathcal{U}_{0}$ to an open set in $G / P$. In particular, $G$ acts on $X_{0}$ with the isotropy subgroup at a generic point isomorphic to $P$, implying $X_{0} \cong G / P$. 


\section{Acknowledgement}

We would like to thank Professor Seok-Jin Kang and Professor Keizo Yamaguchi for providing valuable references.

\section{REFERENCES}

[1] Bourbaki N., Groupes et Algèbres de Lie, Ch. 7-8, Hermann, Paris, 1975.

[2] GRothendieck A., Sur la classification des fibrés holomorphes sur la sphère de Riemann, Amer. J. Math. 79 (1957) 121-138.

[3] HWAng J.-M., Rigidity of homogeneous contact manifolds under Fano deformation, J. Reine Angew. Math. 486 (1997) 153-163.

[4] HWANG J.-M., Stability of tangent bundles of low dimensional Fano manifolds with Picard number 1, Math. Ann. 312 (1998) 599-606.

[5] Hwang J.-M., MoK N., Rigidity of irreducible Hermitian symmetric spaces of the compact type under Kähler deformation, Invent. Math. 131 (1998) 393-418.

[6] HWANG J.-M., MoK N., Holomorphic maps from rational homogeneous spaces of Picard number 1 onto projective manifolds, Invent. Math. 136 (1999) 209-231.

[7] Hwang J.-M., MoK N., Varieties of minimal rational tangents on uniruled projective manifolds, in: Schneider M., Siu Y.-T. (Eds.), Several Complex Variables, MSRI Publications, Vol. 37, Cambridge University Press, 2000, pp. 351-389.

[8] HumphreYs J., Introduction to Lie Algebras and Representation Theory, Grad. Texts Math., Vol. 9, Springer, 1972.

[9] KAC V., Infinite Dimensional Lie Algebras, 3rd Ed., Cambridge University Press, 1990.

[10] Kodaira K., On stability of compact submanifolds of complex manifolds, Amer. J. Math. 85 (1963) 79-94.

[11] Kollár J., Rational Curves on Algebraic Varieties, Ergebnisse Math. 3 Folge, Vol. 32, SpringerVerlag, 1996.

[12] Mok N., Metric Rigidity Theorems on Hermitian Locally Symmetric Manifolds, Series in Pure Math., Vol. 6, World Scientific, 1989.

[13] Morimoto T., Geometric structures on filtered manifolds, Hokkaido Math. J. 22 (1993) 263-347.

[14] Serre J.-P., Complex Semisimple Lie Algebras, Springer-Verlag, 1987.

[15] TANAKA N., On the equivalence problems associated with simple graded Lie algebras, Hokkaido Math. J. 8 (1979) 23-84.

[16] Yamaguchi K., Differential systems associated with simple graded Lie algebras, in: Progress in Differential Geometry, Adv. Study Pure Math., Vol. 22, 1993, pp. 413-494.

Jun-Muk HwANG

Korea Institute for Advanced Study, 207-43 Cheongryangri-dong, Seoul 130-012, South Korea

E-mail: jmhwang@ns.kias.re.kr

Ngaiming MoK

Department of Mathematics, The University of Hong Kong, Pokfulam Road, Hong Kong

E-mail: nmok@hkucc.hku.hk 